\title{
Design and Development of Compact Conformal RFID Antennas Utilizing Novel Flexible Magnetic Composite Materials for Wearable RF and Biomedical Applications
}

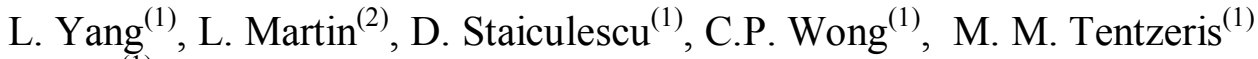 \\ (1) Georgia Institute of Technology, Atlanta, GA, 30332, U.S.A. \\ (2) Motorola, Plantation, FL, 33322, U.S.A.
}

\begin{abstract}
This paper reports the first conformal RFID antenna printed on a flexible magnetic composite material for the UHF frequency band. The target applications are wearable RFIDs and bio-monitoring wireless probes. The successful implementation of the flexible magnetic composite material allows the tag miniaturization and provides excellent electrical performance in the $480 \mathrm{MHz}$ band. First, the material is chosen for optimal electric and magnetic losses, then the antenna is designed for both the non-magnetic material and the magnetic one to prove the miniaturization. Finally, the effect of bending the antenna on the measured return loss performance is investigated.
\end{abstract}

\section{Introduction}

As the technology for RFID systems continuously improves and extends to structures of non-orthogonal shapes and to conformal sensors of wireless body area networks (WBAN), there has been a need to design more "flexible" reader and tag systems. Namely, miniaturization of the transponder and ability to tune the system performance to accommodate EM (electromagnetic) absorption and interference from surrounding media, while compensating for fabrication tolerances has been one of the major priorities [1]. This paper introduces for the first time a novel flexible magnetic composite for printed circuits and antennas, that can reap the same miniaturization and tuning benefits with the heavier and non-flexible 3D used magnetic cores. In previous studies, it can often be cited that the objectives of miniaturization and improved performance are tempered by the limited availability of materials that possess the required magnetic properties, while maintaining an acceptable mechanical and conformality performance [2]. Recently, formulation of nano-size ferrite particles has been reported [3] and formulation of magnetic composites comprised of ferrite filler and organic matrix has been demonstrated [4]. The implication of these new magnetic materials has yet not been investigated for specific EM systems above the low $\mathrm{MHz}$ range. The aim of this work is to provide a basis for this co-design of materials, fabrication processes and electromagnetic structures, namely for the benchmarking case of a novel flexible magnetic composite, a BaCo ferrite-silicone composite, and a UHF RFID antenna, respectively.

Specifically, in this study a benchmark structure was first designed for $480 \mathrm{MHz}$ in a full-wave simulator for an unfilled Silicone substrate; then the magnetic particles were added and the same antenna was redesigned for $480 \mathrm{MHz}$ by reducing its size, thus proving the miniaturization concept. The next step was the actual fabrication of the miniaturized antenna on the magnetic composite and its performance was measured, compared and validated along with the simulated predictions. The presented magnetic substrate is the first flexible magnetic composite tested and proven for the $480 \mathrm{MHz}$ bandwidth with acceptable magnetic losses, that makes it usable for lightweight 
conformal/wearable applications, like pharmaceutical industry and wireless health monitoring in hospital, ambulance and home-based patient care.

\section{Magnetic material development}

The first step in the antenna design was the development of the magnetic composite. The matrix material choice was made for Dow Corning Sylgard 184 silicone. The electrical parameters of the unfilled silicone, used in the initial antenna design, are $\varepsilon_{r}=$ 2.65 and $\tan \delta_{e}=0.001$. The choice for the magnetic composite was Trans-Tech BaCo ferrite powder, product name Co2Z. A 40 vol\% ferrite paste was produced with a mixer at $240 \mathrm{rpm}$ and $110^{\circ} \mathrm{C}$ for 30 minutes. The paste was transferred into a flat mold and vacuum cured with a hold confirmed to occur at $>125^{\circ} \mathrm{C}$ for 50 minutes to produce a 1.3 $\mathrm{mm}$ thick substrate. The material was measured using an HP4291A impedance analyzer to obtain complex permittivity $(\varepsilon)$ and permeability $(\mu)$ (real and imaginary parts) with material fixtures $16453 \mathrm{~A}$ for $\varepsilon$ and $16454 \mathrm{~A}$ for $\mu$ over the frequency range of $1 \mathrm{MHz}$ to 1.8GHz. There were 5 measurements taken for each $\varepsilon_{r}, \mu_{r}, \tan \delta_{e}$ and $\tan \delta_{m}$. Based on these results, the values used in the model were $\varepsilon_{r}=7.14, \mu_{r}=2.46, \tan _{e}=0.0017$ and $\tan \delta_{m}=$ 0.039 .

\section{Antenna design and measurement}

One of the main challenges in designing an RFID tag is the impedance matching between the terminals of the tag antenna and those of the IC. This requires a conjugate matching technique, such as series or parallel stubs and/or using inductively coupling. The matching network of the tag has to guarantee the maximum power delivered to the IC which is used to store the data transmitted to and receive from the RFID reader. The return loss of RFID antenna can be calculated based on the power reflection coefficient which takes into account the reactance part of the IC's impedance

$$
\left|S^{2}\right|=\left|\frac{Z_{I C}-Z_{A N T} *}{Z_{I C}-Z_{A N T}}\right|^{2},
$$

where $Z_{\mathrm{IC}}$ represents the impedance of the IC and $Z_{\mathrm{ANT}}$ represents the impedance of the antenna terminals with $Z_{\mathrm{ANT}}$ *being its conjugate.

Another challenge concerns the dimensions of the RFID tags for flexible/wearable operation. The free space wavelength at $480 \mathrm{MHz}$ is $692 \mathrm{~mm}$. An RFID tag that has the miniaturized features is becoming more of a necessity, for example, in the implementation of an RFID-enable wristband for wireless health monitoring in hospital. To achieve these design goals, a folded bow-tie meander line dipole antenna was designed and fabricated on the characterized magnetic composite material substrate. The RFID prototype structure is shown in Fig. 1 along with dimensions, with the IC placed in the center of the shorting stub arm. The nature of the bow-tie shape of the half wavelength dipole antenna body allows for a broader band operation. The meander line helps realizing further miniaturization of the antenna structure. The shorting stub arm is responsible for the matching of the impedance of the antenna terminals to that of the IC through the fine tuning of the length.

A GS $1000 \mu \mathrm{m}$ pitch probe was used for impedance measurements. In order to minimize backside reflections of this type of antenna, the fabricated antenna was placed on a custom-made probe station using high density polystyrene foam with low relative permittivity of value 1.06 resembling that of the free space. 


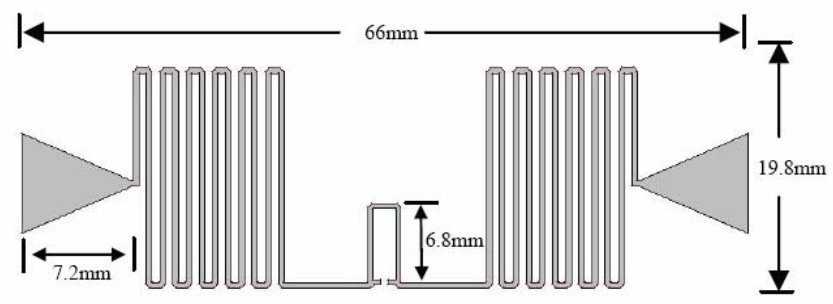

Fig. 1. Configuration of the tag module on magnetic composite substrate.

The calibration method used was short-open-load-thru (SOLT). The initial structure was designed for the lower end of the UHF spectrum and was modeled using Zeland IE3D full wave EM software. The initial substrate was pure silicone $\left(_{r}=2.65\right.$ and $\tan =0.001)$ of $1.3 \mathrm{~mm}$ thickness. Then, the same dimensions of the antenna were maintained for the magnetic composite material. The Return Loss plot is shown in Fig. 2, demonstrating a frequency down shifting of $20 \%$ with increased magnetic permeability, which proves the miniaturization concept. The radiation pattern of the RFID tag module was plotted in Fig. 3. The radiation pattern is almost uniform (omnidirectional) at $480 \mathrm{MHz}$ with directivity around $2.0 \mathrm{dBi}$.

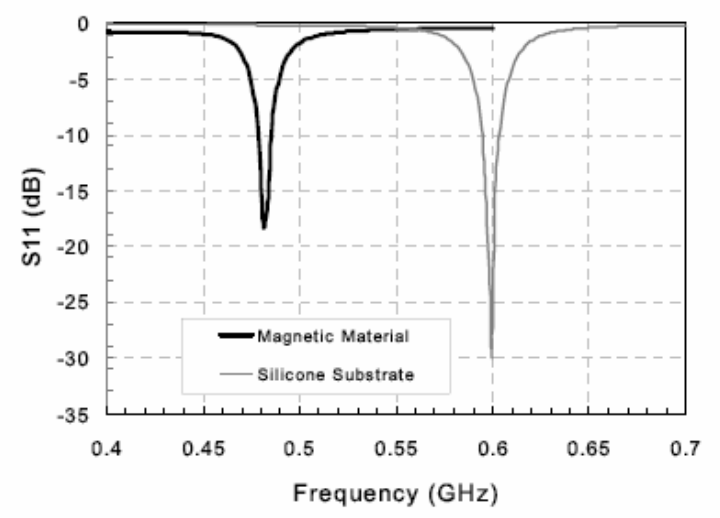

Fig. 2. Simulated return loss (RL) of RFID antenna on magnetic material in comparison with the one on the silicone substrate.
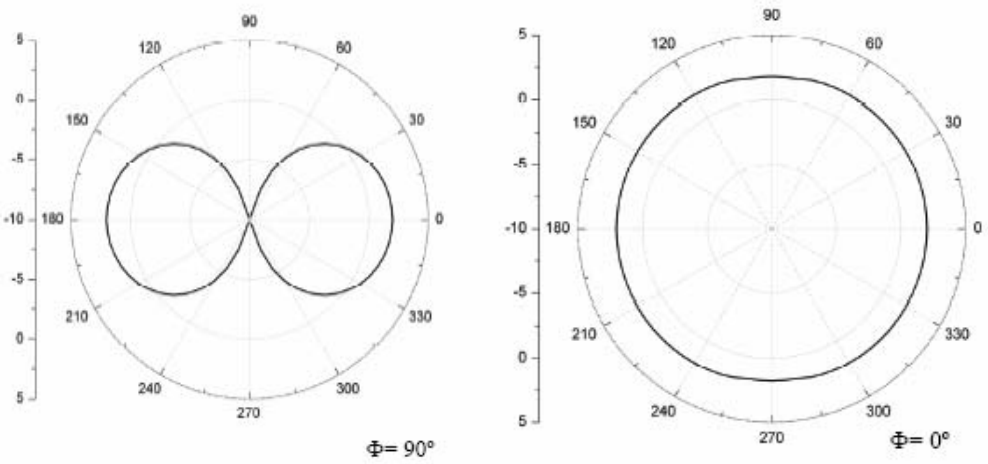

Fig. 3. Simulated 2D far-field radiation pattern plots.

In order to verify the performance of the conformal RFID antenna, measurements were performed as well by sticking the same RFID tag on a foam cylinder with a radius of $54 \mathrm{~mm}$, chosen to resemble a human hand or a drug bottle, as seen in Fig. 4. The return loss results in Fig. 5 show that the return loss of the inkjet-printed antenna is slightly shifted down by $6 \mathrm{MHz}$ with a center frequency at $474 \mathrm{MHz}$. Overall, a very good 
performance is still maintained with the interested band covered. The flexible property of the substrate enables the RFID tag module's application in diverse areas. The conformal RFID tag prototype is usable for wireless health monitoring and pharmaceutical drug bottle tracking, as shown in Fig. 4.

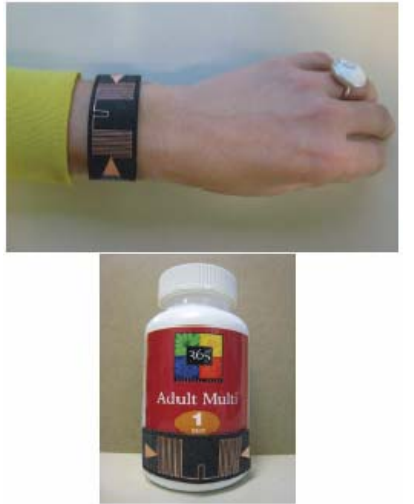

Fig. 4. Medical and pharmaceutical applications

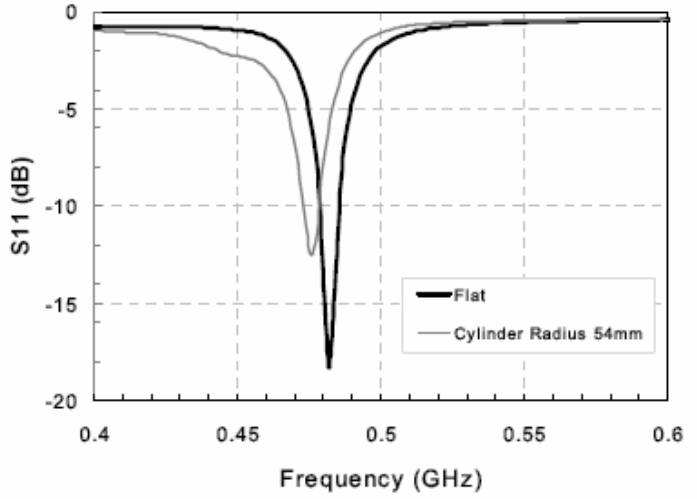

Fig. 5. Measured RL of the flat RFID vs. the conformal tag.

\section{Conclusions}

A combination of electromagnetic tools and measurements has been used to investigate the impact of flexible magnetic composite materials to the miniaturization of RFID antennas. This approach has been applied to the design of a benchmarking conformal RFID tag module and has enabled the assessment of implications that materials have on this design, specifically that the antenna is miniaturized by using the magnetic composite vs. pure silicone. A real composite material has been fabricated and the performance of the miniaturized antenna predicted using the models. This is the first demonstration of a flexible magnetic composite proven for a $480 \mathrm{MHz}$ antenna with acceptable magnetic losses that makes it usable for small size, lightweight conformal applications like wireless health monitoring in pharmaceuticals, hospital, ambulance and home-based patient care.

\section{References}

[1] "Magnetic Materials for RFID," TechnoForum 2005, TDK, http://www.tdk.co.jp/tf2005/pdf e/2f0215e.pdf.

[2] N. Das and A. K. Ray, "Magneto Optical Technique for Beam Steering by Ferrite Based Patch Arrays," IEEE Transactions on Antennas and Propagation, vol. 49, no. 8, August (2001); pp. 1239-1241.

[3] S. Morrison, C. Cahill, E. Carpenter, S. Calvin, R. Swaminathan, M. McHenry, V. Harris, "Magnetic and Structural Properties of Nickel Zinc Ferrite Nanoparticles Synthesized at Room Temperature," Journal of Applied Physics, vol. 95, no. 11, June (2004); pp. 6392-6395.

[4] H. Dong, F. Liu, Q. Song, Z.J. Zhang, C. P. Wong, "Magnetic Nanocomposite for High Q Embedded Inductor," IEEE International Symposium and Exhibition on Advance Packaging Materials: Process, Properties, and Interfaces, Atlanta, Georgia, March (2004); pp. 171-174. 